\title{
ArcheoSciences
}

Revue d'archéométrie

33 (suppl.) | 2009

Mémoire du sol, espace des hommes

\section{Test and data processing of a stepped-frequency GPR array}

\section{Juerg Leckebusch}

\section{(2) OpenEdition \\ 12 Journals}

\section{Electronic version}

URL: https://journals.openedition.org/archeosciences/1751

DOI: 10.4000/archeosciences. 1751

ISBN: 978-2-7535-1599-4

ISSN: 2104-3728

\section{Publisher}

Presses universitaires de Rennes

\section{Printed version}

Date of publication: 30 October 2009

Number of pages: $305-307$

ISBN: 978-2-7535-0943-6

ISSN: $1960-1360$

\section{Electronic reference}

Juerg Leckebusch, "Test and data processing of a stepped-frequency GPR array", ArcheoSciences

[Online], 33 (suppl.) | 2009, Online since 30 October 2011, connection on 21 September 2021. URL: http://journals.openedition.org/archeosciences/1751; DOI: https://doi.org/10.4000/archeosciences. 1751 


\title{
Test and data processing of a stepped-frequency GPR array
}

\author{
Juerg LeCKeBusCH*
}

Key words: GPR, Stepped-frequency, Antenna array, Data processing, Penetration depth.

Recording of ground-penetrating radar data is very time consuming compared to other geophysical prospection methods like magnetics. The spatial resolution of the technique is so high, that a very dense survey grid is necessary to properly record the anomalies and to prevent aliasing. Additionally the antennas need direct contact with the ground, which reduces the maximum possible survey speed in the field. In magnetometry it has become standard to use several sensors to speed up field recording, whereas GPR surveys are done with only one or two antennas. Therefore there is a great potential to reduce field time by using large arrays of GPR antennas. It has been shown, that a profile separation of $25 \mathrm{~cm}$ for a $400 \mathrm{MHz}$ antenna is still too wide for a full waveform recording (Grasmueck et al., 2005). The later is important to get the most detailed information about the underground that is possible to record with GPR. If a large number of antennas are used simultaneously and closely enough, this requirement can be fulfilled too.

For our tests, we used a GeoScope control unit from 3d-radar with a B1831C antenna (Eide and Hjelmstad, 2002). This antenna array consists of 31 antenna element pairs allowing to record 31 profiles in one go. Additionally the system uses a stepped-frequency technique with a very wide range of frequency from $150 \mathrm{MHz}$ to $2000 \mathrm{MHz}$ for the B1831C antenna, hence repeated surveys with different antenna frequencies should no longer be necessary: Theoretically the array covers the spectrum of a 200, 400, $900 \mathrm{MHz}$ and $1.3 \mathrm{GHz}$ antenna from a pulsed system in one trace. But this is difficult to achieve and hence the array is composed of three different antenna element types for low, medium and high frequencies, optimally arranged inside the box. The different type of bow-tie antennas permit using them as air-coupled antennas, making field recording easier. The electronics can acquire lines of CMPs by proper configuring the TX-and RX-elements.

Such a high resolution requires a proper survey in the field including high precision coordinate determination of each single trace. Existing technology using an RTK-GPS or a tracking total station can fulfil these requirements (Leckebusch, 2005b). When an area is covered by such a system, the high density should be maintained throughout the complete area. Hence any gaps between the swaths should be avoided. Using real-time technology and a proper navigation system the data can be recorded with no gaps between the profiles (Fig. 1).

The data that are recorded with a stepped-frequency system are enormous, even for smaller areas. The data from such a system is very different from conventional pulsed radar systems: The information is recorded in frequency domain with a real and imaginary part. Therefore the data must first be converted to time domain. But some processing steps are better applied in the frequency domain before conversion: The system has a very strong ringing and much higher amplitude of the first reflection. These disturbances could be eliminated by a conventional background removal in time domain. Tests have shown that a background remo-

*Terra vermessungen ag. (leckebusch@terra.ch) 


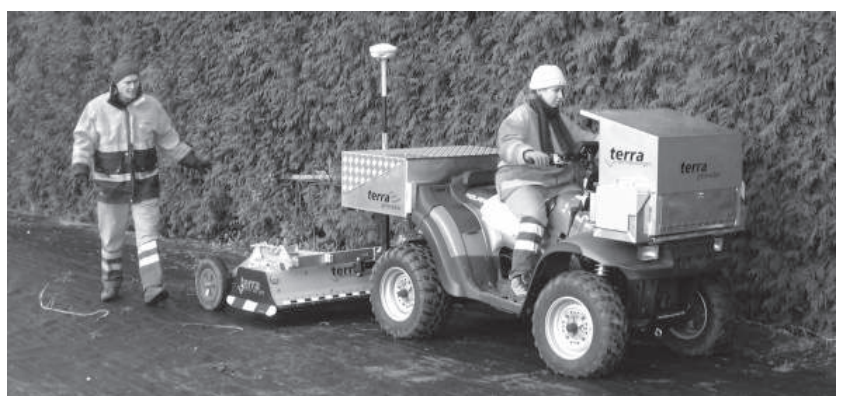

Figure 1: Mounting of the used stepped-frequency GPR array on a Quad bike.

val in frequency domain is more efficient and preserves the reflections much better. There is also a different time delay of the first reflection for each antenna element. Additionally the emission point of the signal varies with frequency (personal communication Egil Eide). Hence the correction of the time delay should be frequency dependent and applied after the background removal, but still in frequency domain. The background removal eliminates also the first reflection because without this process nothing can be seen. Therefore it is very difficult to determine time zero and use any routine to adjust for it. Unfortunately the differences of the amplitudes between adjacent but different antenna elements are high and therefore require a special footprint filter to remove this effect (Leckebusch, 2005a). These datasets are non-gridded compared to traditional surveys. As each trace is provided with $3 \mathrm{D}$ coordinates and the spacing is very close, a full 3D binning and stacking becomes an important part of the data processing. This is necessary at the latest during the calculation of time- or depth-slices.

Proper analysis of the recorded profiles and calculated slices revealed the following: First of all there is a high amplitude horizontal reflection at about $83 \mathrm{~ns}$ (Fig. 2a and b). This seems to be an internal reflection of the system (personal communication Egil Eide) and hence precludes proper data recording in a window of about 75 to $95 \mathrm{~ns}$. Another horizontal, but much weaker reflection at $19 \mathrm{~ns}$ seems to be an internal reflection too. Repeated measurements of the same and very different sites showed, that the system is extremely dependent on the ground conditions and hence coupling of the antenna. In many cases, were a pulsed system delivers excellent results, the array fails even to penetrate the ground. Obviously the coupling is often so bad, that the penetration depth is severely degraded. After trials on archaeological sites, for engineering work, utility location and airport runway surveys, we must conclude, that

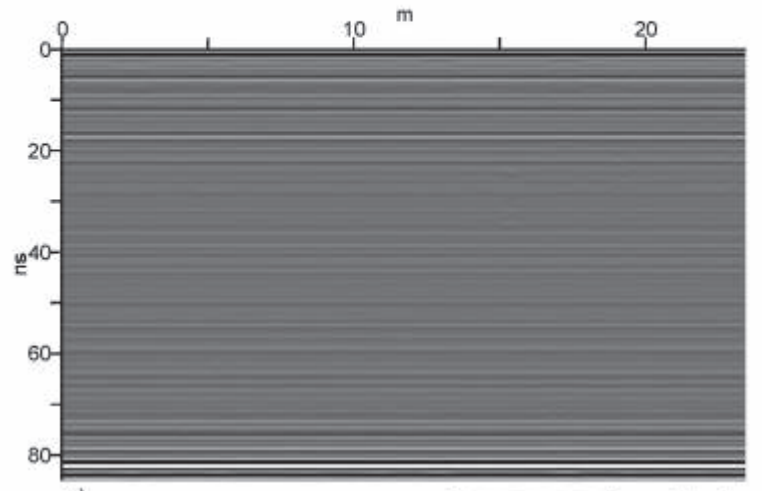

a)

min.

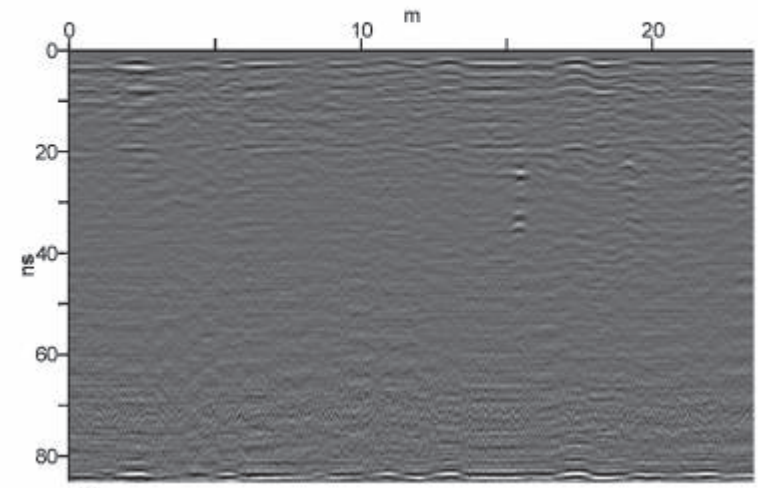

b)

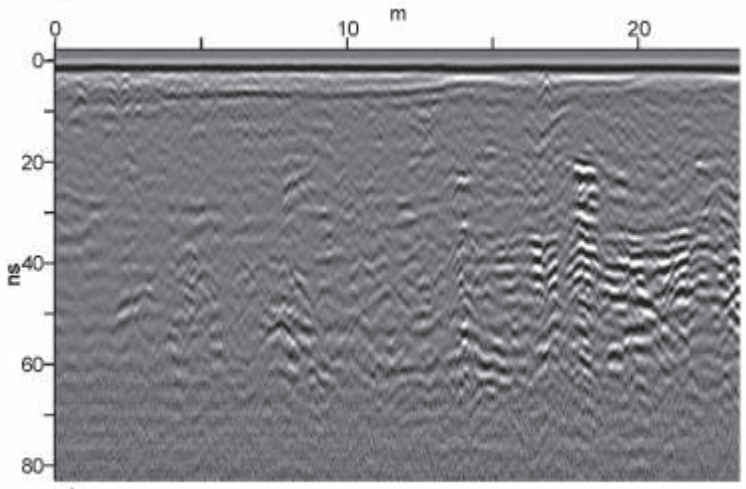

c)
Figure 2: Comparison of a frequency stepped profile 150 - $1000 \mathrm{MHz}$ : a) raw data, b) after background removal of a medium element and c) a raw profile from a conventional pulsed antenna with $400 \mathrm{MHz}$. 

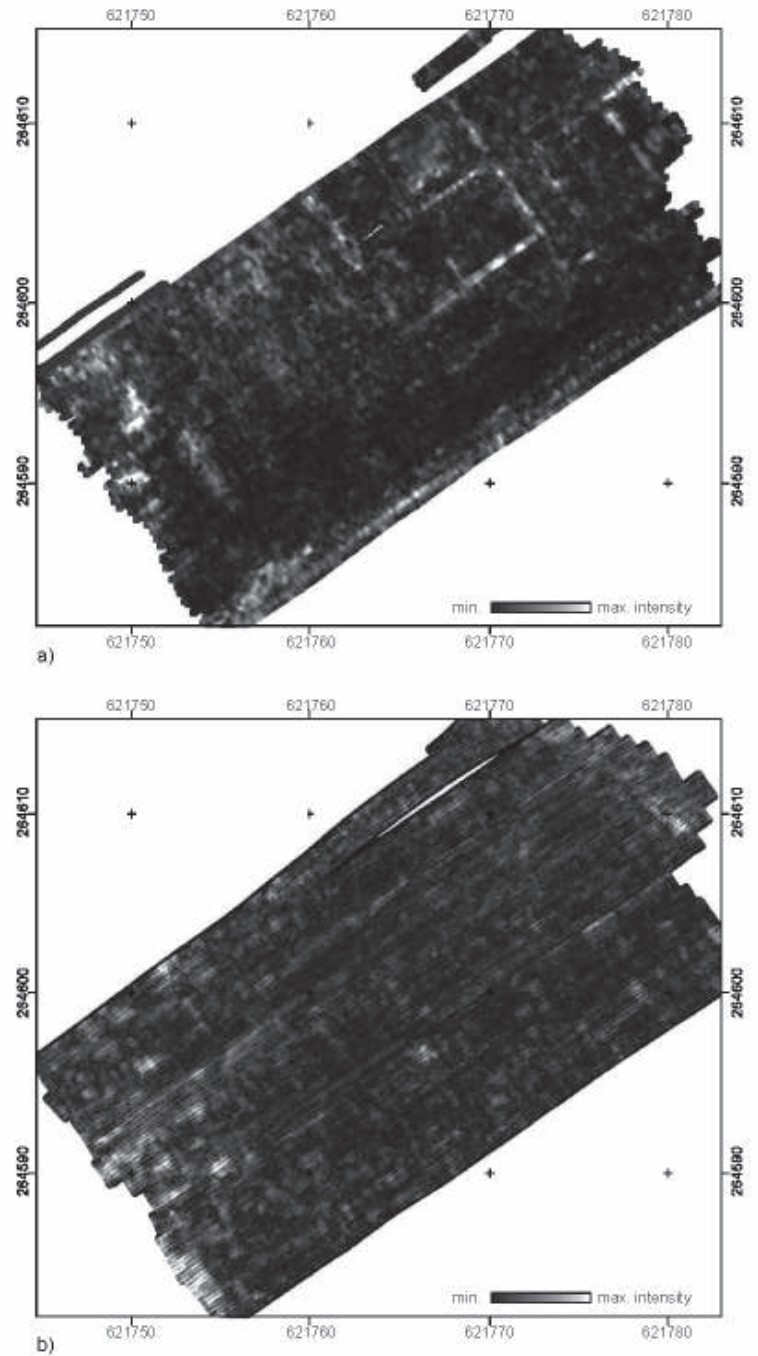

Figure 3: Comparison of depth-slices at a depth of $0.82-0.87 \mathrm{~m}$ recorded with a pulsed a) and a stepped-frequency system b), both datasets without footprint filtering.

the used system is not reliable enough for routine survey. Additionally the time- and depth-slices show high reflection, circular areas, where a pulsed system does not show anything (Fig. 3b) on the left side). These spurious reflections of course cover the underlying archaeological remains. The reason for these remains are unclear.

Hence we must conclude that this frequency-stepped system looks very promising, but cannot be applied during routine surveys. The biggest problem seems to be the use of air-coupled antennas compared to the conventional groundcoupled antennas of pulsed systems.

\section{References}

Eide, E. S. and HJelmstad, J. F., 2002. 3D utility mapping using electronically scanned antenna array. In GPR 2002. Ninth International Conference on Ground Penetrating Radar, Santa Barbara, CA, 192-196.

Grasmueck, M., Weger, R. and Horstmeyer, H., 2005. Fullresolution 3D GPR imaging. Geophysics, 70, (1): K12-K19.

LeCKEBUSCH, J., 2005a. Use of antenna arrays for GPR surveying in archaeology. Near Surface Geophysics, 3, (2): 111-115.

LecKebusch, J., 2005b. Precision real-time positioning for fast geophysical prospection. Archaeological Prospection, 12, (3): 199-202. 